\title{
Functional impairment of the auditory pathway after perinatal asphyxia and the short-term effect of perinatal propofol anesthesia in lambs
}

\author{
Adriana L. Smit ${ }^{1}$, Matthias Seehase ${ }^{2}$, Robert J. Stokroos ${ }^{1,3}$, Reint K. Jellema ${ }^{2}$, Lilian Felipe ${ }^{1}$, Michelene N. Chenault ${ }^{1,3,4}$, \\ Lucien J.C. Anteunis ${ }^{1}$, Bernd Kremer ${ }^{1,5}$ and Boris W. Kramer ${ }^{2,5}$
}

BACKGROUND: Sensorineural hearing loss (SNHL) is a common feature in the postasphyxial syndrome in newborns. Several anesthetic drugs have been proposed to attenuate secondary neuronal injury elicited by hypoxia-ischemia. We hypothesized that propofol anesthesia reduces auditory impairment after perinatal asphyxia in comparison with isoflurane.

METHODS: Twenty-three pregnant ewes were randomized to propofol or isoflurane anesthesia and sedation. The lambs underwent in utero umbilical cord occlusion (isoflurane $n=5$; propofol $n=7$ ) and were compared with sham-treated animals (isoflurane $n=5$; propofol $n=6$ ) at a gestational age of 133 d. For $8 \mathrm{~h}$ after delivery by cesarean section, repeated auditory brainstem responses (ABRs) were recorded to obtain hearing thresholds, peak amplitudes, latencies, and interpeak latencies. RESULTS: Significantly elevated mean thresholds, diminished amplitudes, and elevated latencies were observed in the asphyxia group relative to the control group through the observation period. Comparison of anesthetic treatment in the asphyxia group revealed a significantly lower elevation in threshold and less impairment in the ABR amplitudes and latencies during propofol anesthesia as compared with isoflurane anesthesia.

CONCLUSION: Our results support the hypothesis that anesthesia with propofol has a preventive effect on the functional changes to the auditory pathway in the event of perinatal asphyxia.

ongenital sensorineural hearing loss (SNHL) is common. In the Western world, the incidence is $1-3$ per 1,000 live births (1-4). In the neonatal intensive care population, the incidence is even higher, with 2-4 per 100 live births (3). Perinatal hypoxic-ischemic insults, which occur in 1-6 per 1,000 live full-term births, are one of the major risk factors for auditory impairment (5-7) and severe neurological sequelae in newborn infants (8). Experimental studies suggest that there are already direct negative effects on hearing parameters within several hours to days after a peri- or postnatal event of severe hypoxia-ischemia (9-11).

To date, little is known regarding the possibilities for intervention in this process. Several agents have been proposed to attenuate secondary neuronal injuries elicited by hypoxia, including anesthetic drugs (12). Given the fact that each drug administered in a neonatal intensive care setting is associated with additional side effects for the newborn, the advantage of anesthetic drugs consists of their unavoidable need of administration in a situation after resuscitation and mechanical ventilation. This raises the question of whether it is possible to prevent or attenuate damage to the auditory pathway in asphyctic newborns by administering the right anesthetic drug.

Previous in vivo and in vitro studies demonstrated neuroprotective effects of several anesthetic drugs in hypoxicischemic insults $(13,14)$. Of these, propofol is one of the most promising agents in mediating neuroprotection after hypoxicischemic insults in adult patients and animals (15-18). However, in the developing brain, anesthetic agents have also been shown to induce apoptosis in newborn animal models (19). So far, it is not known if propofol exerts a protective or aversive effect to the auditory pathway in perinatal asphyxia-induced functional hearing impairment. By comparing the effect of propofol, which exerts its effect via gamma aminobutyric acid and $N$-methyl-Daspartate receptors (20), with isoflurane anesthesia, which acts via sodium channel inhibition (21), we tried to gain insight into protective strategies to diminish asphyxia-induced functional damage to the auditory pathway.

We hypothesized that (i) perinatal hypoxic-ischemia results in SNHL and (ii) perinatal propofol anesthesia reduces hypoxia-ischemia-induced SNHL as compared with isoflurane anesthesia. To test this hypothesis, late-preterm instrumented sheep fetuses were exposed to in utero umbilical cord occlusion (UCO) at $133 \mathrm{~d}$ of gestation (term: $150 \mathrm{~d}$ ) (22-24). Fetal sheep of this gestational age are more susceptible to

\footnotetext{
The first two authors contributed equally to this work.

'Department of Otorhinolaryngology/Head and Neck Surgery, Maastricht University Medical Centre, Maastricht, The Netherlands; ${ }^{2}$ Department of Pediatrics, Maastricht University Medical Centre, Maastricht, The Netherlands; ${ }^{3}$ School of Mental Health and Neuroscience, University of Maastricht, Maastricht, The Netherlands; ${ }^{4}$ Department of Methodology and Statistics, Maastricht University Medical Centre, Maastricht, The Netherlands; ${ }^{5}$ School of Oncology and Developmental Biology, University of Maastricht, Maastricht, The Netherlands. Correspondence: Adriana L. Smit (diane.smit@mumc.nl)
} 
neurological damage after UCO than mid-gestation fetal sheep according to antenatal drug choice (25). After delivery, fetuses were anesthetized with either propofol or isoflurane. Mothers received the same anesthetic drug during instrumentation and UCO of their fetus. The functional integrity of the auditory brainstem pathway was assessed by repeated auditory brainstem response (ABR) recordings of hearing threshold, peak amplitudes, latencies, and interpeak latencies during $8 \mathrm{~h}$ of observation.

\section{RESULTS}

\section{Physiological Outcome}

Observations were made in all 23 fetal sheep at a mean gestational age of $133.8 \pm 0.18 \mathrm{~d}$. The groups did not differ according to gestational age, gender, or birth weight $(3.5 \pm 0.1 \mathrm{~kg})$. The total UCO time needed until the mean fetal arterial blood pressure (MABP) dropped below $30 \mathrm{~mm} \mathrm{Hg}$ for 2 min was not significantly different between the isoflurane group $(11.3 \pm 0.6 \mathrm{~min})$ and the propofol group $(10.7 \pm 0.3 \mathrm{~min})$. All animals developed bradycardia with a frequency of $<30 / \mathrm{min}$ after UCO and needed resuscitation. The $\mathrm{pH}$ before birth was similar in all four groups $(7.28 \pm 0.02)$. The $\mathrm{pH}$ after birth decreased to $6.92 \pm 0.05$ in the propofol asphyxia group as compared with a $\mathrm{pH}$ of $7.15 \pm 0.04$ in the propofol control group. In the isoflurane group, the $\mathrm{pH}$ after birth was $6.88 \pm 0.02$ in the asphyxia group as compared with $7.11 \pm 0.02$ in the control group. In the asphyxia group, no significant differences were detected between anesthesia groups in MABP (isoflurane: 61.3 \pm 6.2 mm Hg vs. propofol: $66.1 \pm 7.3 \mathrm{~mm} \mathrm{Hg} P=0.300$ ) or heart rate (isoflurane: $187.4 \pm 7.9 \mathrm{bpm}$ vs. propofol: $181.6 \pm 16.2 \mathrm{bpm} ; P$ $=0.493)$. There was a significant difference in the used dose of propofol $(P<0.001)$ and isoflurane $(P<0.001)$ between the control and asphyxia groups (Table 1$)$.

\section{ABR Recordings}

$A B R$ recordings were made in all lambs. Of the five positive ABR peaks, the first peak tended to coincide with the stimulus artifact at a click intensity of $40 \mathrm{~dB}$ above the ABR threshold (Figure 1). Wave III was the most prominent waveform peak in the ABR pattern, in contrast to a small peak IV, which was situated alongside the downhill slope of peak III.

\section{Hearing Threshold}

The mean threshold was overall in time significantly higher in the asphyxia group than in the control group

Table 1. Dose of anesthetics used in lambs

\begin{tabular}{ll}
\hline & $\begin{array}{c}\text { Propofol } / \text { isoflurane } \\
\text { dose }\end{array}$ \\
\hline Propofol control & $1.68 \pm 0.08 \mathrm{mg} / \mathrm{kg} / \mathrm{h}^{*}$ \\
Propofol asphyxia & $1.14 \pm 0.06 \mathrm{mg} / \mathrm{kg} / \mathrm{h}$ \\
Isoflurane control & $0.86 \pm 0.02 \% *$ \\
Isoflurane asphyxia & $0.57 \pm 0.02 \%$ \\
\hline
\end{tabular}

Data are given as mean \pm SEM. $P$ values compare control vs. asphyxia per anesthetic treatment (control vs. asphyxia). ${ }^{*} P<0.001$. (control: $28.6 \pm 1.4 \mathrm{~dB}$ peak-equivalent sound pressure level vs. asphyxia: $32.7 \pm 1.1 \mathrm{~dB}$ peak-equivalent sound pressure level; $P=0.034$ ). This difference was consistent by comparison of the individual time intervals. Differentiation in the asphyxia group between the anesthetics showed a significantly lower threshold in the propofol than in the isoflurane group $(P=0.013)$ (Table 2$)$. The mean threshold was inversely correlated to both the $\mathrm{pH}$ (Pearson correlation coefficient: $-0.34 ; P=0.026$ ) and the MABP (Pearson correlation coefficient: $-0.30 ; P=0.049$ ).

\section{Amplitudes}

Peak amplitudes showed significantly lower values in the asphyxia group as compared with the control group for peaks II (control: $0.280 \pm 0.022 \mu \mathrm{V}$ vs. asphyxia: $0.182 \pm 0.020 \mu \mathrm{V} ; P=$ 0.003 ), III (control: $0.852 \pm 0.034 \mu \mathrm{V}$ vs. asphyxia: $0.653 \pm 0.034$ $\mu \mathrm{V} ; P<0.001$ ), and V (control: $0.144 \pm 0.015 \mu \mathrm{V}$ vs. asphyxia: $0.127 \pm 0.013 \mu \mathrm{V} ; P=0.035)$ after correction for anesthetic treatment. The amplitude of peak IV was too small to allow reliable recording. In the asphyxia group, differentiation according to anesthetic treatment demonstrated significantly lower mean amplitude for peak III after isoflurane sedation in comparison with propofol sedation $(P=0.038)$ during the total time of observation. Statistically significant differences between anesthetic treatments in the asphyxia or control group were not seen for the other peak amplitudes (Table 2).

\section{Latencies}

Significantly higher mean absolute latencies were seen after asphyxia as compared with the control group for peaks III (control: $4.683 \pm 0.070 \mathrm{~ms}$ vs. asphyxia: $5.064 \pm 0.053 \mathrm{~ms}$; $P<0.001$ ) and V (control: $7.870 \pm 0.081 \mathrm{~ms}$ vs. asphyxia: $8.288 \pm 0.052 \mathrm{~ms} ; P<0.001)$. For peak II, the same tendency was observed, but this was not statistically significant $(P=$ 0.052). These findings were consistent throughout the time intervals. Differentiation to anesthetic treatment in the asphyxia group showed significantly less elevated latency times in the propofol group than in the isoflurane group for peaks III $(P=0.015)$ and V $(P=0.046)$ (Table 2$)$. In the control group, no significant differences were observed between anesthetic treatments.

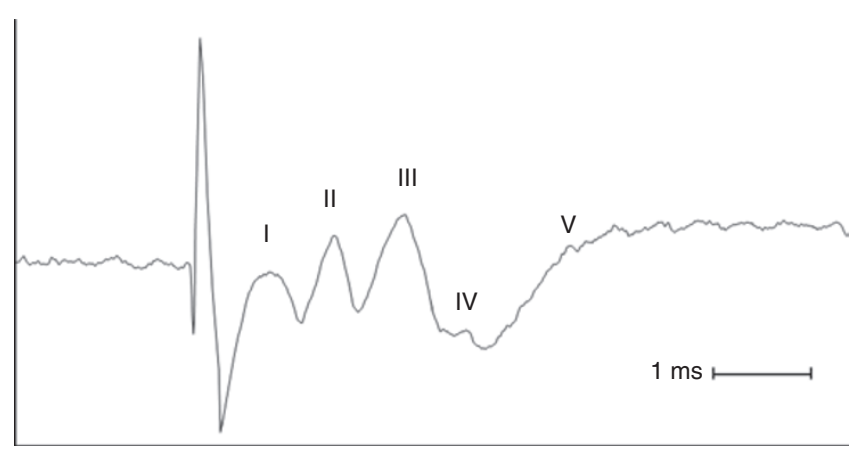

Figure 1. Bone-conducted stimulus ABR waveform pattern in the lamb model of $133 \mathrm{~d}$ of gestational age. The markers identify the waveform peaks $(I-V)$ that were used for analysis. ABR, auditory brainstem response. 


\section{Articles | Smitet al.}

Table 2. ABR parameters of waveform peaks according to interventions and treatment

\begin{tabular}{|c|c|c|c|c|c|c|}
\hline & \multirow[b]{2}{*}{ Peak } & \multicolumn{2}{|c|}{ Control } & \multicolumn{2}{|c|}{ Asphyxia } & \multirow{2}{*}{$\begin{array}{c}\text { Control vs. } \\
\text { asphyxia } \\
P\end{array}$} \\
\hline & & Isoflurane $(n=5)$ & Propofol $(n=6)$ & Isoflurane $(n=5)$ & Propofol $(n=7)$ & \\
\hline \multirow[t]{2}{*}{ Amplitude } & 1 & $0.240(0.027)$ & $0.223(0.025)$ & $0.192(0.026)$ & $0.202(0.024)$ & NS \\
\hline & II & $0.279(0.043)$ & $0.281(0.012)$ & $0.152(0.030)$ & $0.220(0.018)$ & $* *$ \\
\hline \multirow[t]{4}{*}{ Latencies } & I & $2.098(0.071)$ & $2.068(0.072)$ & $2.136(0.054)$ & $2.149(0.049)$ & NS \\
\hline & ॥ & $3.232(0.095)$ & $3.245(0.057)$ & $3.407(0.075)$ & $3.380(0.061)$ & NS \\
\hline & III & $4.709(0.103)$ & $4.658(0.099)$ & $5.177(0.069)$ & $4.930^{\dagger}(0.065)$ & $\S$ \\
\hline & IV & $7.162(0.117)$ & $7.075(0.119)$ & $7.172(0.067)$ & $7.199(0.070)$ & NS \\
\hline Interpeak latencies & $\mathrm{I}-\mathrm{V}$ & $5.757(0.110)$ & $5.769(0.139)$ & $6.078(0.049)$ & $5.980(0.092)$ & * \\
\hline
\end{tabular}

Mean hearing threshold (dB peSPL), amplitudes ( $\mu \mathrm{V}$ ), latencies (ms), and interpeak latencies (ms) per peak (interval) according to sham control or asphyxia group and anesthetic treatment. Data are given as mean \pm SEM. Comparison of isoflurane vs. propofol treatment within the control or asphyxia group $\left({ }^{\dagger} P<0.05\right)$. $P$ values compare control vs. asphyxia after correction for difference in anesthetic treatment (control vs. asphyxia).

ABR, auditory brainstem response; NS, nonsignificant; peSPL, peak-equivalent sound pressure level.

${ }^{*} P<0.05$. ${ }^{*} P<0.01 .{ }^{\S} P<0.001$.

\section{Interpeak Intervals}

Mean interpeak latencies of intervals I-V were significantly higher in the asphyxia group than in the control group (control: $5.763 \pm 0.087 \mathrm{~ms}$ vs. asphyxia: $6.033 \pm 0.050 \mathrm{~ms} ; P=0.016$ ) and were consistent during the whole time of observation. After differentiation to anesthetic treatment, no significant differences were seen between isoflurane and propofol sedation after correction for time interval in the asphyxia or control group (Table 2).

\section{DISCUSSION}

SNHL is a common feature in the postasphyxial syndrome. In this study, we showed the effect of perinatal asphyxia on the auditory brainstem parameters and the impact of intervention by ante- and postnatal propofol vs. isoflurane anesthesia in a late-preterm lamb model.

Analysis of the ABR waveforms during the first $8 \mathrm{~h}$ after severe perinatal asphyxia demonstrated a direct impairment of the ABR parameters, which was consistent throughout the time of observation. In this period, a statistically significant elevated threshold was observed after asphyxia as compared with the sham-treated control group. However, the magnitude of the observed differences in threshold is small and around the precision of the testing procedure, with $5 \mathrm{~dB}$ steps. These effects could be more obvious in a more severe event of umbilical cord compression, which was shown to be feasible in the lamb model (25). Nevertheless, the described effect of threshold impairment was supported by impaired peak amplitudes and latencies of earlier and later components of the auditory pathway. The presented results correlate with existing literature describing the effects of asphyxia on hearing function.
Gunn et al. (26) demonstrated prolongation of latencies and decreased amplitudes within hours to days after severe hypoxemia with acidosis in a fetal lamb model. Jiang et al. $(9,10)$ confirmed this outcome in human term neonates. These functional data together are in line with the findings of brainstem lesions with neuronal necrosis at sites concerning the auditory pathway after an event of perinatal asphyxia (27).

To gain insight into protective strategies to diminish perinatal asphyxia-induced functional damage to the auditory pathway, we compared propofol and isoflurane anesthesia. In our experiment, we found a significantly smaller impairment of the ABR threshold after propofol as compared with isoflurane anesthesia. This protective effect of propofol was also present for the observed alterations of the amplitude of peak III and latencies of peaks III and V. Although the described effects are small, these findings were reproducible throughout the time of observation.

The neuroprotective effects of propofol after hypoxicischemic insults in adult animals have been proposed to be attributable to several mechanisms, including free-radical scavenging (28), potentiation of gamma aminobutyric acidmediated inhibition of synaptic transmission, and inhibition of $N$-methyl-D-aspartate-type glutamate receptor currents (29-32). Which mechanism is responsible for the observed effects in our model of auditory effects in late-preterm lambs cannot be elucidated here.

A limitation of the current study is the short time of observation of the lambs after the insult, resulting from need for intensive care with ventilation and sedation. By this, a potential spontaneous (partial) recovery of auditory function in days to weeks after the hypoxic-ischemic insult, as described by Jiang et al. $(9,10)$, could not be studied. Second, by administering the 
anesthetic drugs before birth to the maternal-fetal unit during cesarean section and postnatally to the newborns, we tried to imitate the situation during emergency cesarean section. However, with this approach, we cannot differentiate between the effect of propofol during or directly after the asphyctic event.

Our results support the hypothesis of a protective effect to the auditory function in lambs by antenatal anesthesia with propofol to prevent the negative effects of perinatal asphyxia. However, in the developing brain of newborn animals, propofol has also been shown to potentiate apoptotic neurodegenerative effects (33-35). Whether this will outweigh the positive auditory outcome by propofol anesthesia must be considered when designing neuroprotective strategies to prevent auditory impairment after perinatal asphyxia.

\section{METHODS}

\section{Study Design}

This study was performed according to the guidelines of the Animal Care Committee of the University of Maastricht, which approved the protocol. Twenty-three time-mated Texel ewes and the preterm fetuses of both genders at a gestational age of $133 \mathrm{~d}$ were used. They were randomized according to an event of asphyxia by UCO in utero or sham procedure. Second, they were randomized for anesthetic/ sedative treatment by propofol (sham: $n=6$; asphyxia: $n=7$ ) or isoflurane (sham: $n=5$; asphyxia: $n=5$ ).

\section{Procedures}

As previously reported (36), pregnant ewes underwent cesarean section under general anesthesia by thiopental induction $(15 \mathrm{mg} / \mathrm{kg})$ and isoflurane anesthesia $(1-2 \%)$ or propofol both as induction $(6 \mathrm{mg} / \mathrm{kg})$ and general anesthesia $(25 \mathrm{mg} / \mathrm{kg} / \mathrm{h})$. In both groups, anesthesia was supplemented by continuous remifentanyl infusion $(3 \mu \mathrm{g} / \mathrm{kg} / \mathrm{min})$. After having opened the uterus, the fetus was endotracheally intubated, and a vascular occluder was placed around the umbilical cord (OC16HD, $16 \mathrm{~mm}$; In Vivo Metric, Healdsburg, CA). UCO was timed until the MABP dropped below $30 \mathrm{~mm} \mathrm{Hg}$ for $2 \mathrm{~min}$. This resulted in severe bradycardia (heart rate: $<30 \mathrm{bpm}$ ) or a complete cardiac arrest. Sham-treated animals underwent the same surgical procedures, except for the actual UCO. After delivery, the fetus was resuscitated according to current guidelines (37), ventilated, and sedated either with propofol or isoflurane. After delivery, analgesia was obtained with intravenous remifentanyl $(3 \mu \mathrm{g} / \mathrm{kg} / \mathrm{min})$, and sedation was maintained with propofol $(1-3 \mathrm{mg} / \mathrm{kg} / \mathrm{h})$ or isoflurane $(0.5-1 \%)$ for $8 \mathrm{~h}$. The dosage was based on the clinical condition. The animals were killed at the end of the experiment with a lethal dose of intravenous T61 (Veterinaria AG, Zurich, Switzerland). Arterial and venous lines were used for continuous monitoring of MABP and heart rate and for repetitive hourly blood sampling to obtain $\mathrm{pH}$ values (Abbott i-STAT 1 Blood Gas Analyzer; Abbott Laboratories, Abbott Park, IL).

\section{ABR Recording Protocol}

During the $8 \mathrm{~h}$ of observation, hourly recordings were conducted using an ABR device (Synergy version 14.1; Viasys Healthcare, Surrey, UK). The ABR waveform was elicited by a bone-conducted click-evoked stimulus of $0.1 \mathrm{~ms}$ per click, with a repetition rate of 11.7 clicks $/ \mathrm{s}$ and alternating polarity. The conductor was fixed on the forehead with an elastic strap to ensure proper contact. A subcutaneous needle electrode was placed on the mastoid, and a reference and a ground electrode were placed on the nasal bridge in the midline. To elicit ABR wave morphology, responses were passed through a $100-3,000 \mathrm{~Hz}$ filter. Impedance of the electrodes was kept below $6 \mathrm{~K} \Omega$. A thousand individual responses to the clicks were averaged for each run (range: 1,000-1,008). Threshold was determined by lowering the intensity in a $10 \mathrm{~dB}$ down, $5 \mathrm{~dB}$ up procedure until responses disappeared. The minimal intensity stimulus to which an $\mathrm{ABR}$ response was evident was considered as the threshold and expressed as $\mathrm{dB}$ peak-equivalent sound pressure level.
Measurements of ABR variables (peak latencies, amplitudes, and interpeak intervals) were assessed at a click intensity of $40 \mathrm{~dB}$ above the ABR threshold of each subject. In all cases, replicate comparisons confirmed test-retest reliability of all traces. Two independent blinded observers trained in ABR recording techniques completed the identification of all parameters visually. Peaks of waveforms that appeared consistently within and across subjects were serially numbered (I-V) based on the recording of vertex upward deflections (Figure 1) (38). The peak was graphically defined by the intersection of lines extended from the positive and negative slopes of the peaks. Because of a sloping aspect of peak $\mathrm{V}$ with an absent distinct negative through after the peak, the amplitude of peak $\mathrm{V}$ was calculated from the peak to the negative through following peak III. Absolute latency of a peak was referenced to stimulus onset. Interpeak latencies were determined from the respective peak latencies. If the peaks did not occur at the same latency on the replicate tracings, the $\mathrm{ABR}$ was considered to have low test-retest reliability, and the data were not used.

\section{Statistical Analyses}

Statistical calculations were made using SPSS 15.0 for Windows (SPSS, Chicago, IL). ABR parameter measurements were grouped according to time interval to facilitate comparison of the effects of interventions in time (time interval I: $0-3$ h, interval II: $3-5$ h, interval III: $5-8 \mathrm{~h}$ after birth). Independent sample $t$-tests and repeated-measures ANOVA analyses over the three time intervals were applied to compare the effects of intervention and treatments. $P$ values of $<0.05$ were considered statistically significant. Data are given as mean \pm SEM.

\section{STATEMENT OF FINANCIAL SUPPORT}

The study was supported by a grant from the Heinsius Houbolt Foundation and the Foundation of Pediatrics, Maastricht University Medical Centre.

Disclosure: The authors declared no conflict of interest.

\section{REFERENCES}

1. Mencher GT. Challenge of epidemiological research in the developing world: overview. Audiology 2000;39:178-83.

2. Davis A, Wood S, Healy R, Webb H, Rowe S. Risk factors for hearing disorders: epidemiologic evidence of change over time in the UK. J Am Acad Audiol 1995;6:365-70.

3. Erenberg A, Lemons J, Sia C, Trunkel D, Ziring P. Newborn and infant hearing loss: detection and intervention. American Academy of Pediatrics. Task Force on Newborn and Infant Hearing, 1998-1999. Pediatrics 1999;103:527-30.

4. Nance WE, Lim BG, Dodson KM. Importance of congenital cytomegalovirus infections as a cause for pre-lingual hearing loss. J Clin Virol 2006;35:221-5.

5. Borg E. Perinatal asphyxia, hypoxia, ischemia and hearing loss. An overview. Scand Audiol 1997;26:77-91.

6. Pierrat V, Haouari N, Liska A, Thomas D, Subtil D, Truffert P; Groupe d'Etudes en Epidémiologie Périnatale. Prevalence, causes, and outcome at 2 years of age of newborn encephalopathy: population based study. Arch Dis Child Fetal Neonatal Ed 2005;90:F257-61.

7. de Haan M, Wyatt JS, Roth S, Vargha-Khadem F, Gadian D, Mishkin M. Brain and cognitive-behavioural development after asphyxia at term birth. Dev Sci 2006;9:350-8.

8. Johnston MV, Trescher WH, Ishida A, Nakajima W. Neurobiology of hypoxic-ischemic injury in the developing brain. Pediatr Res 2001;49: 735-41.

9. Jiang ZD, Yin R, Shao XM, Wilkinson AR. Brain-stem auditory impairment during the neonatal period in term infants after asphyxia: dynamic changes in brain-stem auditory evoked response to clicks of different rates. Clin Neurophysiol 2004;115:1605-15.

10. Jiang ZD, Brosi DM, Wang J, et al. Time course of brainstem pathophysiology during first month in term infants after perinatal asphyxia, revealed by MLS BAER latencies and intervals. Pediatr Res 2003;54:680-7.

11. Inagaki M, Kaga M, Isumi $H$, Hirano $S$, Takashima $S$, Nanba E. Hypoxiainduced ABR change and heat shock protein expression in the pontine auditory pathway of young rabbits. Brain Res 1997;757:111-8. 
12. Schifilliti D, Grasso G, Conti A, Fodale V. Anaesthetic-related neuroprotection: intravenous or inhalational agents? CNS Drugs 2010;24:893-907.

13. Koerner IP, Brambrink AM. Brain protection by anesthetic agents. Curr Opin Anaesthesiol 2006;19:481-6.

14. Kotani Y, Shimazawa M, Yoshimura S, Iwama T, Hara H. The experimental and clinical pharmacology of propofol, an anesthetic agent with neuroprotective properties. CNS Neurosci Ther 2008;14:95-106.

15. Acquaviva R, Campisi A, Raciti G, et al. Propofol inhibits caspase-3 in astroglial cells: role of heme oxygenase-1. Curr Neurovasc Res 2005;2:141-8.

16. Chen L, Xue Z, Jiang H. Effect of propofol on pathologic time-course and apoptosis after cerebral ischemia-reperfusion injury. Acta Anaesthesiol Scand 2008;52:413-9.

17. Hans P, Bonhomme V. Why we still use intravenous drugs as the basic regimen for neurosurgical anaesthesia. Curr Opin Anaesthesiol 2006;19:498503.

18. Vasileiou I, Xanthos T, Koudouna E, et al. Propofol: a review of its nonanaesthetic effects. Eur J Pharmacol 2009;605:1-8.

19. Istaphanous GK, Loepke AW. General anesthetics and the developing brain. Curr Opin Anaesthesiol 2009;22:368-73.

20. Irifune M, Takarada T, Shimizu Y, et al. Propofol-induced anesthesia in mice is mediated by gamma-aminobutyric acid-A and excitatory amino acid receptors. Anesth Analg 2003;97:424-9, table of contents.

21. Durrmeyer X, Vutskits L, Anand KJ, Rimensberger PC. Use of analgesic and sedative drugs in the NICU: integrating clinical trials and laboratory data. Pediatr Res 2010;67:117-27.

22. Back SA, Riddle A, Hohimer AR. Role of instrumented fetal sheep preparations in defining the pathogenesis of human periventricular white-matter injury. J Child Neurol 2006;21:582-9.

23. Hagberg H, Peebles D, Mallard C. Models of white matter injury: comparison of infectious, hypoxic-ischemic, and excitotoxic insults. Ment Retard Dev Disabil Res Rev 2002;8:30-8.

24. Wolfs TG, Jellema RK, Turrisi G, Becucci E, Buonocore G, Kramer BW. Inflammation-induced immune suppression of the fetus: a potential link between chorioamnionitis and postnatal early onset sepsis. J Matern Fetal Neonatal Med 2012;25:Suppl 1:8-11.

25. Mallard EC, Williams CE, Johnston BM, Gluckman PD. Increased vulnerability to neuronal damage after umbilical cord occlusion in fetal sheep with advancing gestation. Am J Obstet Gynecol 1994;170(1 Pt 1): 206-14.
26. Gunn AJ, Cook CJ, Williams CE, Johnston BM, Gluckman PD. Electrophysiological responses of the fetus to hypoxia and asphyxia. J Dev Physiol 1991;16:147-53.

27. Pasternak JF. Hypoxic-ischemic brain damage in the term infant. Lessons from the laboratory. Pediatr Clin North Am 1993;40:1061-72.

28. Grasshoff C, Gillessen T. The effect of propofol on increased superoxide concentration in cultured rat cerebrocortical neurons after stimulation of N-methyl-d-aspartate receptors. Anesth Analg 2002;95:920-2, table of contents.

29. Orser BA, Bertlik M, Wang LY, MacDonald JF. Inhibition by propofol (2,6 di-isopropylphenol) of the N-methyl-D-aspartate subtype of glutamate receptor in cultured hippocampal neurones. Br J Pharmacol 1995;116:1761-8.

30. Abboud TK, Zhu J, Richardson M, Peres Da Silva E, Donovan M. Intravenous propofol vs thiamylal-isoflurane for caesarean section, comparative maternal and neonatal effects. Acta Anaesthesiol Scand 1995;39:205-9.

31. Wakasugi M, Hirota K, Roth SH, Ito Y. The effects of general anesthetics on excitatory and inhibitory synaptic transmission in area CA1 of the rat hippocampus in vitro. Anesth Analg 1999;88:676-80.

32. Buonocore G, Perrone S, Turrisi G, Kramer BW, Balduini W. New pharmacological approaches in infants with hypoxic-ischemic encephalopathy. Curr Pharm Des 2012;18:3086-100.

33. Bercker S, Bert B, Bittigau P, et al. Neurodegeneration in newborn rats following propofol and sevoflurane anesthesia. Neurotox Res 2009;16:140-7.

34. Pesic V, Milanovic D, Tanic N, et al. Potential mechanism of cell death in the developing rat brain induced by propofol anesthesia. Int J Dev Neurosci 2009;27:279-87.

35. Fredriksson A, Pontén E, Gordh T, Eriksson P. Neonatal exposure to a combination of N-methyl-D-aspartate and gamma-aminobutyric acid type A receptor anesthetic agents potentiates apoptotic neurodegeneration and persistent behavioral deficits. Anesthesiology 2007;107:427-36.

36. Seehase M, Houthuizen P, Jellema RK, et al. Propofol administration to the fetal-maternal unit reduces cardiac injury in late-preterm lambs subjected to severe prenatal asphyxia and cardiac arrest. Pediatr Res 2013;73:427-34.

37. Vento M, Saugstad OD. Resuscitation of the term and preterm infant. Semin Fetal Neonatal Med 2010;15:216-22.

38. Pierson LL, Gerhardt KJ, Griffiths SK, Abrams RM. Auditory brainstem response in sheep. Part I: fetal development. Dev Psychobiol 1995;28:293305. 